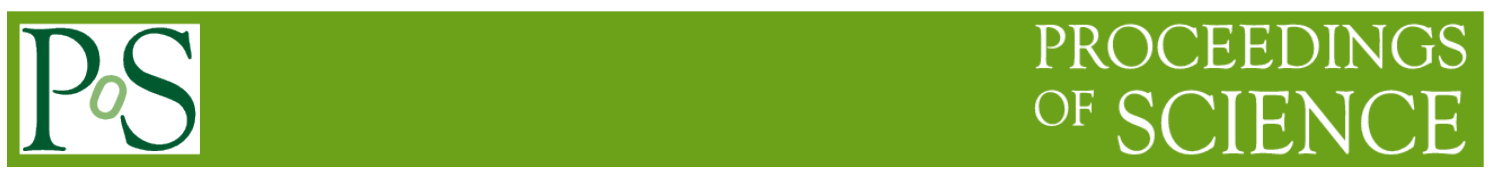

\title{
The Magnetic Universe
}

\section{Marijke Haverkorn}

Fac. NWI, Radbout University Nijmegen, Postbus 9010, 6500 GL Nijmegen, The Netherlands E-mail:m.haverkorn@astro.ru.nl

\section{Michiel Brentjens}

ASTRON

Oude Hoogeveensedijk 4, 7991 PD Dwingeloo, The Netherlands

E-mail: brentjens@astron.nl

50 Years Westerbork Radio Observatory, A Continuing Journey to Discoveries and Innovations Richard Strom, Arnold van Ardenne, Steve Torchinsky (eds) 


\title{
Chapter 9 The Magnetic Universe
}

\author{
Marijke Haverkorn', Michiel Brentjens²
}

\section{Introduction}

Radio emission has opened a new window on the Universe. Radio polarization opens yet another. It is the interaction with magnetic fields that polarizes radio waves, and properties of this magnetic field are imprinted in the polarization signature. Hence, radio polarization is one of the few methods to study elusive, largely invisible, cosmic magnetic fields.

Magnetic fields are hugely important in space, in many objects and on many scales. Most of the (baryonic) matter in space is ionized, which means that the gas is closely coupled to magnetic fields, often almost frozen into them. Magnetic fields influence star formation: by delaying it in the first stages, and stabilizing (through magnetic braking) in the last phase. Magnetic fields cause acceleration of cosmic rays, and guide their transport across the Universe. Magnetism is the origin of the Solar cycle and Solar activity. The Earth's magnetic field protects us from harmful radiation from space, and magnetic fields around exoplanets are thought to increase the habitability on those planets.

One of the main physical processes emitting radio waves in space is synchrotron radiation, which is emitted by electrons moving at velocities close to the speed of light, while spiraling around magnetic field lines. The intensity of this radiation depends on the magnetic field strength and direction, integrated over the line of sight. Synchrotron intensity is intrinsically highly linearly polarized perpendicular to the direction of the local magnetic field component in the plane of the sky. However, if magnetic fields vary along the line of sight, the orientation of polarization angle varies as well, which results in partial depolarization. Therefore, the degree of polarization indicates the degree of tangling of the magnetic field.

A second, equally versatile, method that uses radio polarization to measure magnetic fields is Faraday rotation. Faraday rotation is the effective rotation of the linear polarization angle as a function of frequency, caused by the birefringence in their circular polarization while radio waves propagate through an

1 Radbout University Nijmegen, The Netherlands 2 ASTRON, The Netherlands ionized, magnetized medium. The amount of rotation is called the Rotation Measure (RM), which depends on the free electron density in the medium, and the local magnetic field component parallel to the line of sight, integrated over that line of sight. Therefore, measuring linear polarization as a function 
of frequency allows the calculation of the RM. Using known models for the free electron density, we can calculate the weighted, average, magnetic field strength along the line of sight. A method to calculate RMs correctly in complex situations, called RM Synthesis, was proven to be applicable in practice by Michiel Brentjens and Ger de Bruyn, using WSRT observations as the proof of concept (Brentjens \& de Bruyn, 2005, A\&A, 441, 1217).

The WSRT is an excellent instrument to measure synchrotron radiation, including its angle and degree of polarization. Its short spacings allow a high sensitivity to extended, diffuse structures such as galaxy cluster gas, Galactic objects such as supernova remnants, and diffuse Galactic synchrotron emission. The excellent $6 \times 12 \mathrm{hr}$ uv-coverage and parallactic mounts made achieving high dynamic range and high image fidelity relatively easy. In particular, the wideband multi-channel MFFE receivers at $92 \mathrm{~cm}$ were a unique tool to derive weaker magnetic fields and exploit RM Synthesis to the fullest.

In this chapter, we will briefly highlight some of the scientific contributions that the WSRT has made to discover the diffuse polarized Universe, with emphasis on the large role that Ger de Bruyn has played in this. We will close with a brief outlook to the immediate future for polarization with the WSRT.

\section{Galaxy clusters}

Clusters of galaxies are the largest gravitationally bound structures in the universe. These clusters can contain hundreds to thousands of galaxies, embedded in a huge cloud of hot thermal gas. Radio emission is commonly observed in clusters: emission from the cluster galaxies themselves, non-thermal emission from the cosmic rays centered at the cluster center - called radio halos - and non-thermal emission from the outskirts of the cluster - called radio relics. These radio relics are elongated, sometimes remarkably long and straight, and highly polarized. They are thought to indicate the location of huge shock waves, created in a past merger of two smaller clusters.

Roberto Pizzo, then PhD student with Ger de Bruyn, used the WSRT to study galaxy cluster Abell 2255 at a wide range of wavelengths from $18 \mathrm{~cm}$ to $2 \mathrm{~m}$. the latter being the wavelength range where LOFAR is now producing many exciting results on this topic. He was the first to detect a galaxy cluster with the WSRT using the newly available technique of RM Synthesis (see Figure 1). He studied Faraday rotation from the hot gas inside the cluster (the intracluster medium), and showed that known radio filaments are highly-polarized radio relics at the outskirts of the cluster.

Subsequent WSRT polarization work mostly focused on radio relics, producing magnificent results due to the exquisite sensitivity and polarization characteristics of these measurements. From detailed mapping of the variation of the synchrotron emission across the relics, it as been possible to gain knowledge about the location and process of particle acceleration at those shocks. The po-

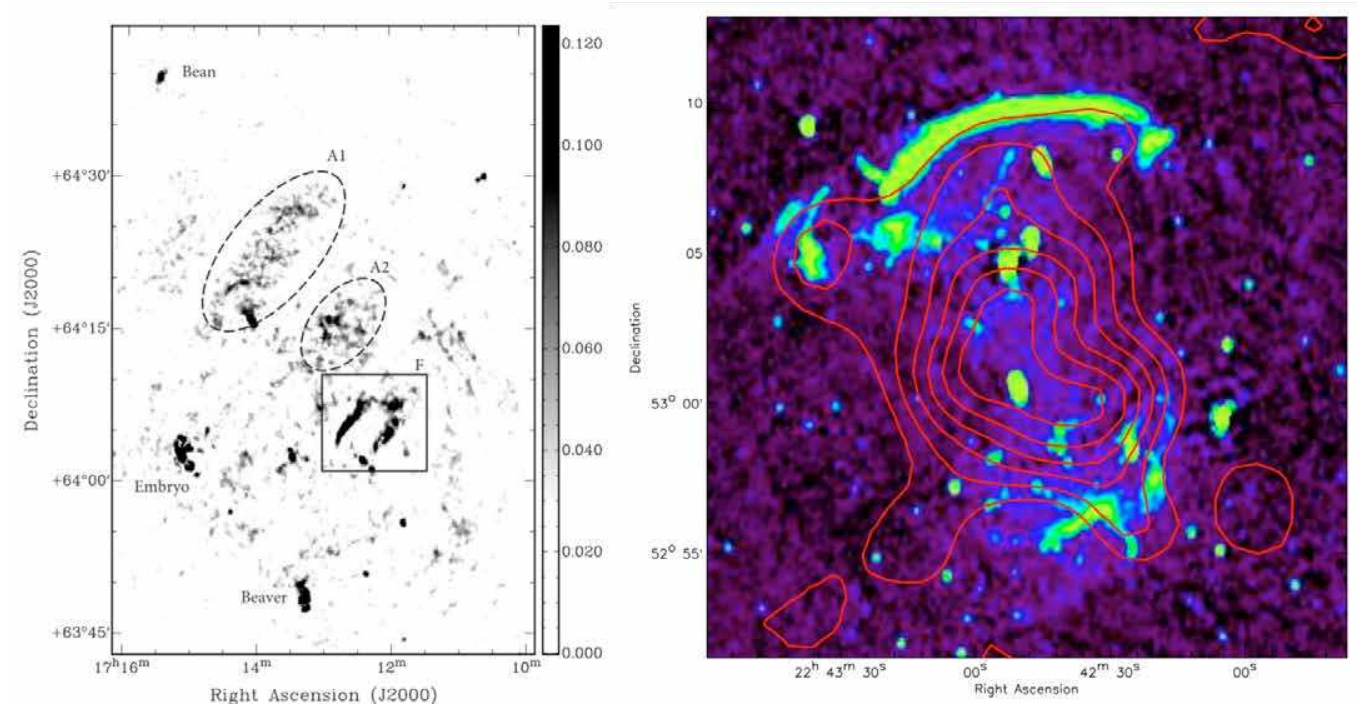

larization in these filaments complements these observations by revealing the Figure 1: Left: One strength and direction of the magnetic field. Magnetic fields are aligned parallel frame from a WSRT to the relic, as expected for a shock wave, and depolarization or polarization $3 D$ RM Synthesis angle directions can be used to infer information on the relative location of the data cube of the relic inside the cluster system. The image shows cluster galaxies and large-scale filaments, that share Radio galaxies and quasars

Radio galaxies are a special, prevalent type of galaxy in the Universe. The centers of these galaxies, which can be spiral or elliptical galaxies, host an active supermassive black hole. This black hole sucks in matter from its surroundings through a rotating disk around it. This process causes huge magnetized jets of matter and radiation to be expelled in either direction of the galaxy. At the distance where they significantly interact with diffuse, intergalactic gas surrounding the galaxy, these jets fan out to become large-scale structures called lobes (see Figure 2).

Both jets and lobes are strong non-thermal (synchrotron) emitters in the radio band. Much work on interpreting their polarization characteristics has been done with the WSRT, primarily in the 8os and 9os. Ger de Bruyn pioneered this starting with his PhD thesis work on Seyfert galaxies. At that time, he obtained only non-detections of linear polarization, giving upper limits of a few percent to up to $\sim 15 \%$ linear polarization.

The degree of polarization is often interpreted as a measure of small-scale fluctuations in density and/or magnetic field. Polarimetric surveys of a large number of (mostly unresolved) radio sources show fairly low polarization degrees, indicating that radio galaxies live in a magnetized (depolarizing) environment. 
Figure 2: Image of the typical radio galaxy DA 240 WSRT radio observations at $50 \mathrm{~cm}$ (magenta and red) show the central source, jets and lobes. VLITE radio obsenations at gocm (blue) show the large extension of the lobes. Optical data (green, DSS shows the central galaxy and background sources. Credn. Produced al the U.S. Naval Research Laboratory by Dr. S. Ciacintucci and collaborators from data obtained with the VLA Low-band Ionosphere and Transient Experiment (VLITE).

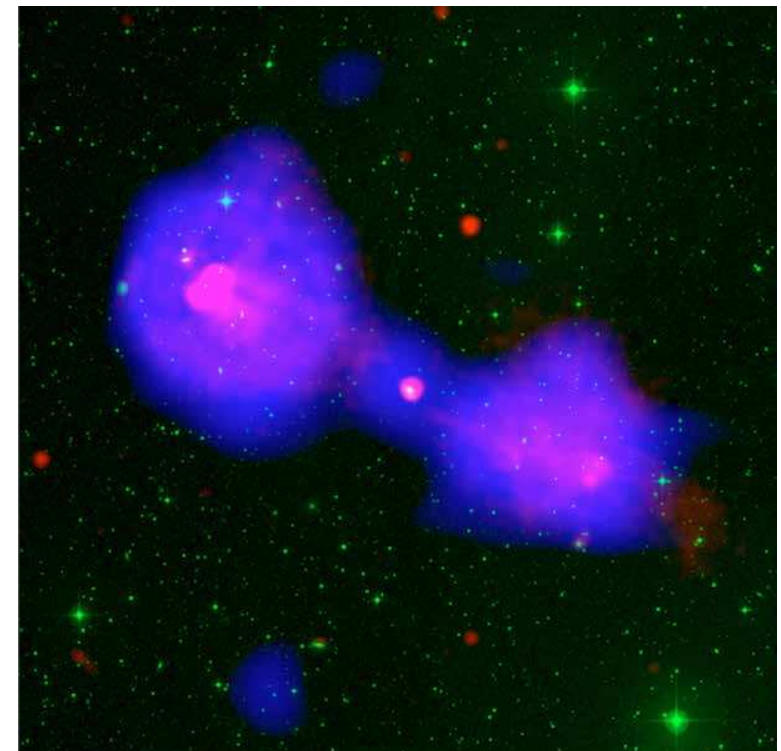

This can be compared to independent density estimates from the assumption that the lobes would be in ram-pressure equilibrium with their surroundings. The polarization vectors (corrected for Faraday rotation at low frequncies) indicate the orientation of the magnetic field component in the plane of the sky. An example of this is given in Figure 3, which shows electric-field vectors and the magnetic field configuration derived from WSRT 1.4 GHz data across the lobes of radio galaxy B2 $0326+39$. Work by Arno Schoenmakers, then PhD student Figure 3: Left: E-field vector orientations $1.4 \mathrm{CHz}$ WRT observations of radio galaxy $\mathrm{B}_{2} 0326+39$ Right: implied orientations of magnetic field after correction for Faraday rotation (Bridle et al 1991, ACXA, 245, 371)
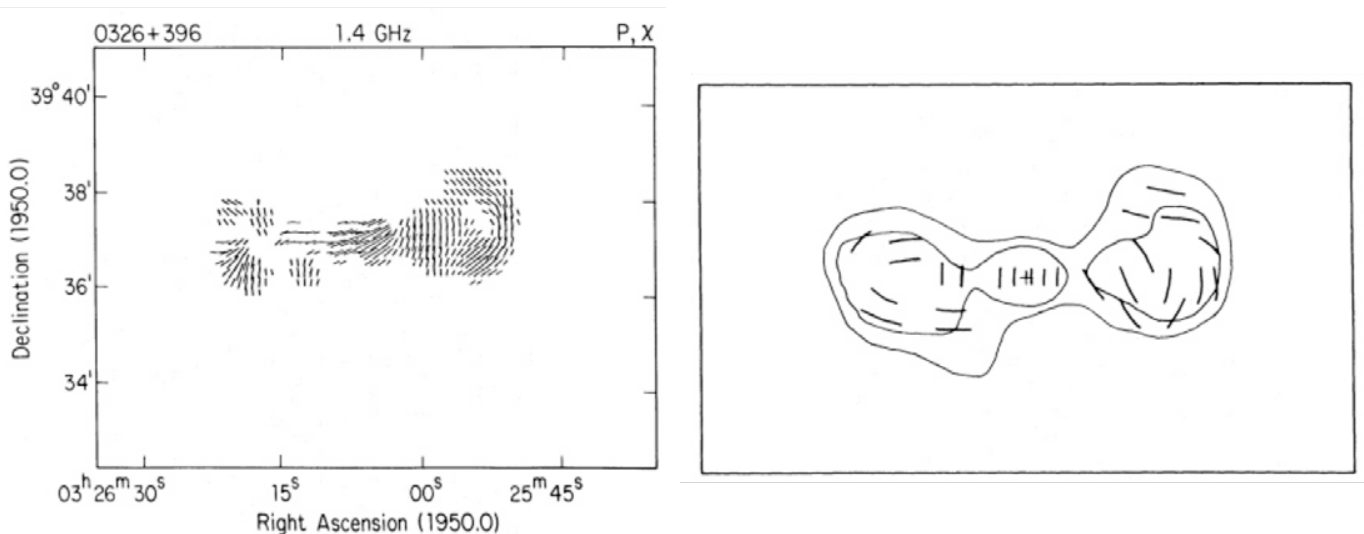
supervised by Ger de Bruyn and Harry van der Laan, also indicates that typically, magnetic fields in (FRII) radio lobes are observed to be tangential to the lobe boundaries. This agrees with the theory that magnetic fields are compressed in outward moving shocks due to interaction with the surrounding intergalactic medium. Weak radio sources tend to have jets with transverse magnetic fields, as pictured here, whereas in more powerful radio sources, magnetic fields tend to be parallel to the sources (Feretti et al 1983, A\&A, 126, 311)
Many studies have measured the Faraday rotation in the central galaxy, jets and/or lobes of radio galaxies. Using traditional RM determination makes it often hard to distinguish whether the observed Faraday rotation is due to a medium in or near the radio galaxy, or due to foreground magnetized gas in the Milky Way. RM values have been interpreted as Faraday curtains in fron of the radio sources, containing irregular magnetic fields and/or a clumpy medium (Rossetti et al 2008, A\&A, 487, 865). However, RM Synthesis in 585 unresolved radio sources revealed the existence of multiple RM components in many sources, rendering earlier RM determinations potentially unreliable (Farnsworth et al 2011, AJ, 141, 191).

\section{Normal galaxies}

Our Milky Way is just one in many spiral galaxies, consisting of a thin disk with stars and gas mostly contained in spiral arms, and a halo of more diffuse gas and fewer stars around it. Studying nearby spiral galaxies in detail gives a picture of the variety in spiral galaxies, but also their common properties. These studies help to understand our own Galaxy: since we are immersed in the Milky Way, it is hard to see the global structure. External spirals provide us with the overview picture we are missing for the Milky Way.

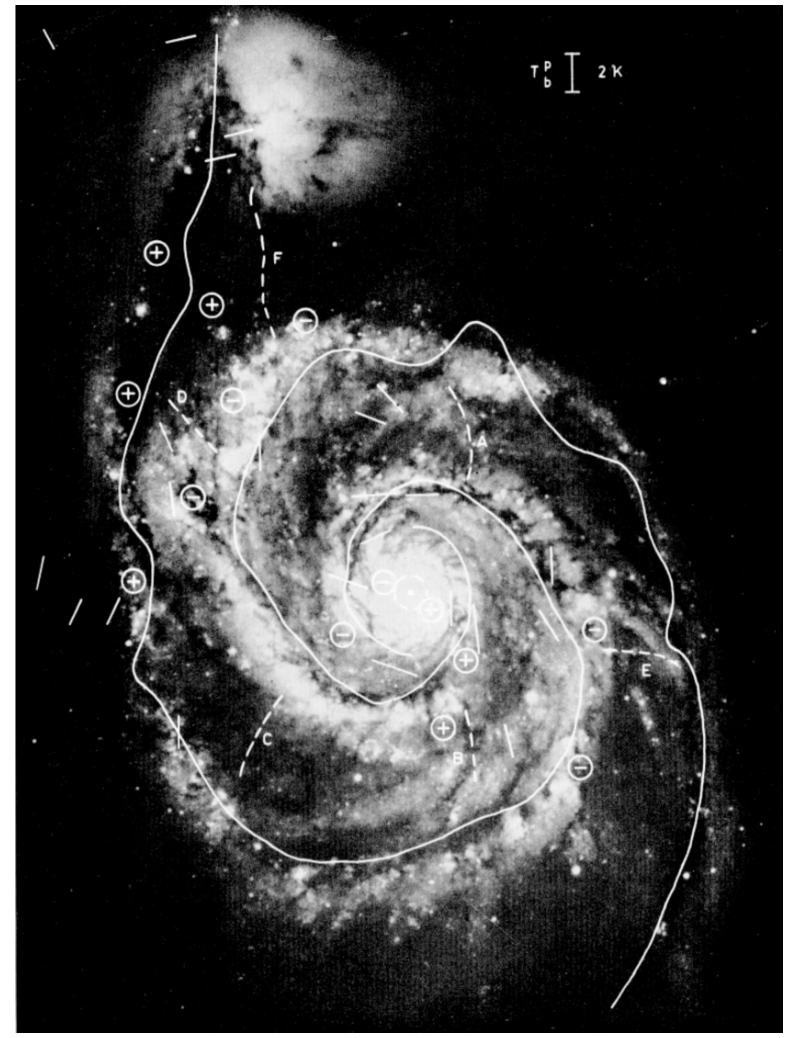

Figure 4: The first tentative detection nearby galaxy M51, made by the WSRT. The long solid lines denote. solid lines denote
ridges of $1415 \mathrm{MHz}$ radio emission, and radio emission, and indicate the E-vectors of the polarized radio emission

The length of the

The length of the

polarized brightness

temperature. 
The WSRT has pioneered polarization measurements in nearby galaxies by a first tentative detection of polarization in nearby grand-design spiral galaxy M51 (Mathewson et al, 1972, A\&A, 17, 468, see Figure 4). Since then, it has been used extensively to map out magnetic fields and polarization properties of nearby galaxies in detail. Ger de Bruyn's PhD thesis also contains the first attempts to detect polarization in spiral galaxies with the WSRT, again resulting in only upper limits. He continued work in this area with his PhD student Alexander Segalovitz, who solidly confirmed linear polarization detections in M51 and concluded that magnetic fields in its disk are oriented preferably tangentially (Segalovitz et al, 1976, Nature, 264, 222). Since that time, the WSRT has mapped magnetic fields in spiral galaxies such as the Milky Way, in interacting galaxies, dwarf galaxies, starburst galaxies and irregular galaxies.

In particular, the magnetic field configuration in halos of galaxies, away from the star-forming disk, is important as it provides clues about the dynamo mechanism that maintains these fields, and may be dynamically important for the gas kinematics (see Chapter 11). The low-frequency MFFE receivers on the WSRT have played a major role in determining weak magnetic fields in galaxy halos; however, follow-up investigations at a broad range of frequencies are essential to obtain the complete picture.

The largest survey of polarization properties of nearby galaxies with the WSRT is its follow-up of the Spitzer Infrared Nearby Galaxies Survey (SINGS) galaxies, named "Westerbork SINGS" (Braun et al 2007, A\&A, 461, 455). In 21 of the 28 SINGS galaxies, polarized emission was detected and RM Synthesis was applied. Coherent depolarization and RM patterns led to a model of corkscrew-magnetic field configurations in the halos of these galaxies (Braun et al 2010, A\&A, 514, 42, Heald et al 2009, A\&A, 503, 409). In M31, diffuse polarized emission from a nearby galaxy below $1 \mathrm{GHz}$ was observed for the first time (Giessuebel et al 2013, A\&A, 559, 27). After this proof of concept, LOFAR continues to map out nearby galaxies with exquisite sensitivity at low frequencies.

\section{Diffuse Galactic radiation}

In the late 1980's, PhD student Mark Wieringa, supervised by Peter Katgert and Ger de Bruyn, saw something unexpected in his WSRT radio data of far-away radio galaxies when he looked at the polarization maps. He discovered large diffuse structures of many degrees in size in polarized emission, which were not visible in total (unpolarized) emission. Their analysis of this emission in terms of synchrotron emission in the Milky Way, Faraday rotated by the Galactic magnetized interstellar medium, started a new avenue for investigating Galactic magnetism. Peter and Ger continued this with a follow-up PhD project, by Marijke Haverkorn, which focused completely on observations and interpretation of these enigmatic polarized structures. Since then, large polarized structures have been found in every field on the sky that was investigated. At lower latitudes, some of these structures are aligned with the Galactic plane,

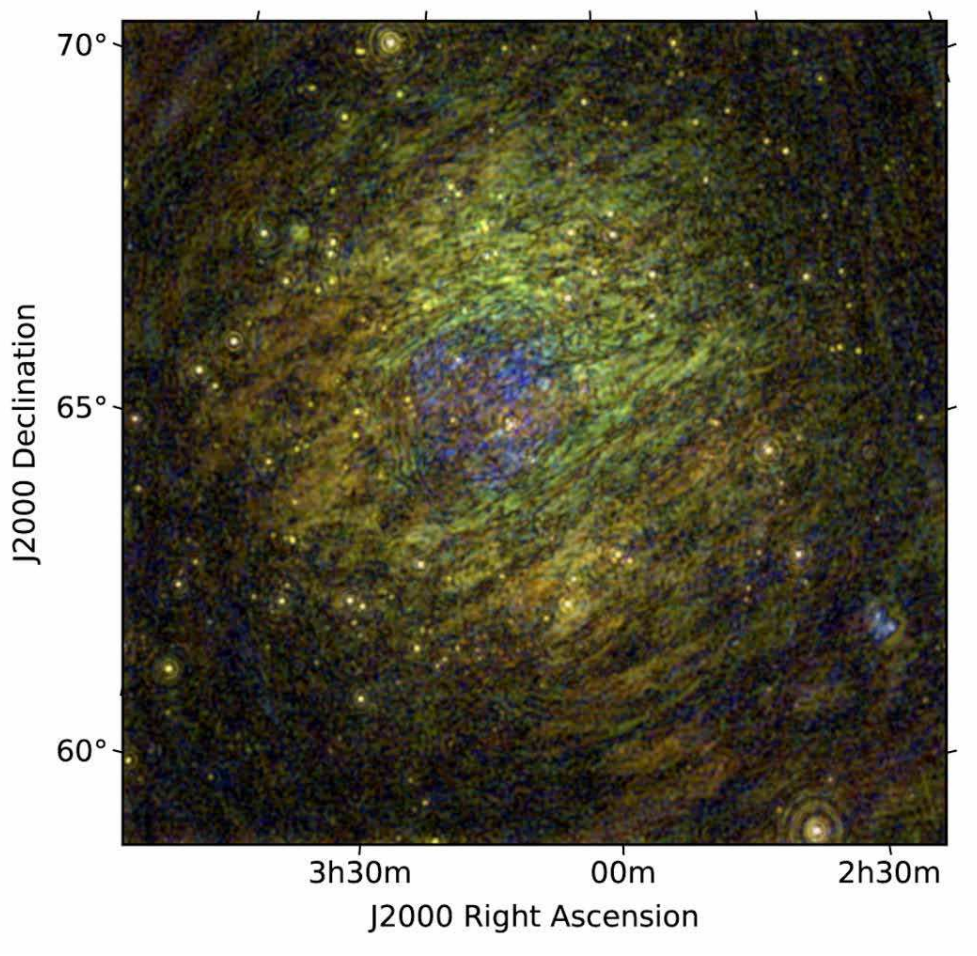

ized intensity at ized intensity at depths (a proxy for density-weighted magnetic field) in magnetized bubble in the Fan Region. (Bernardi et al 2009 AबA, 500, 965; color scale optimized to mimic response of human eye by Michiel Brentjens.

suggesting a directionality along the regular magnetic field in the plane. Ger de Bruyn initiated polarimetric analysis of parts of the Westerbork Northern Sky Survey (WENSS, see Chapter 10), demonstrating the ubiquity and coherence of these polarized structures (Schnitzeler et al, 2007, A\&A, 461, 963). Enigmatic bubbles (e.g. Figure 5), filaments, depolarization canals and other odd-shaped structures have been detected, showing us magnetic field features otherwise undetectable. The WSRT has played a pioneering role in early interpretation of these structures, most notably in early RM Synthesis results and in the broad long-wavelength coverage when combined with LOFAR. Much of this effort was led by PhD students Gianni Bernardi and Vibor Jelic in Ger de Bruyn's group. A recent exciting discovery -- amongst others in WSRT data --is the correlation of such synchrotron polarization structures with magnetic fields as probed by Planck polarized dust emission (Zaroubi et al 2015, MNRAS, 454 46) or by filaments of neutral hydrogen (Kalberla \& Kerp, 2016, A\&A, 595, 37).

\section{The Future}

The upgrade of the WSRT to the Apertif Focal Plane Array system will enable exploring broadband spectropolarimetry at frequencies of 1-2 $\mathrm{GHz}$, which un fortunately meant largely decommissioning the $92 \mathrm{~cm}$ system. At the Apertif frequency band, the sensitivity for low-RM signal will decrease, but high RMs will become detectable, and the polarized source density on the sky will 
be higher than at lower frequencies. The largest planned polarization survey with Apertif is the Westerbork Observations of the Deep Apertif Northern-Sky (WODAN) survey. The many 100,000's of expected detections of polarized radio sources across the sky will provide a detailed RM Grid with which to test Galactic magnetic field models. In addition, the polarized diffuse Galactic emission will allow determination of the properties of the small-scale structure in this field. In this way, the WSRT will stay at the forefront of cosmic magnetism science for many years to come.

\section{Acknowledgements}

The authors thank Rainer Beck, George Heald, Arno Schoenmakers, and Reinout van Weeren for their valuable input. 